\title{
Derecho y vida: \\ un contrapunto entre Michel Foucault y Giorgio Agamben
}

\author{
Law and life: \\ a Counterpoint between Michel Foucault and Giorgio Agamben
}

Edgardo Castro*

\begin{abstract}
Resumen: Desde la perspectiva de la crítica al antijuridicismo foucaultiano expuesta por Giorgio Agamben en Homo sacer, en el presente trabajo abordamos la cuestión del derecho y su relación con la vida en la genealogía de la racionalidad política moderna, desarrollada en los cursos de Michel Foucault en el Collège de France de los años 19781979. Nuestro objetivo es mostrar las modalidades que adopta esta relación a través de los conceptos de integración marginalista y de utilitarismo político, de golpe de Estado permanente y de heterogeneidad de las formas jurídicas. A partir de estas nociones, nos proponemos, además, una confrontación con la lectura realizada por Agamben.
\end{abstract}

Palabras clave: Integración marginalista, utilitarismo político, biopolítica, tanatopolítica, formas jurídicas.

Abstract: From the perspective of the criticism of Foucaultian antijuridicism presented by Giorgio Agamben in Homo sacer, in this paper we address the question of law and its relation to life in the genealogy of modern political rationality, developed in the courses of Michel Foucault in the Collège de France from the years 1978-1979. Our goal is to show the modalities that this relationship adopts through the concepts of marginalistic integration and political utilitarianism, permanent coup d'état and heterogeneity of legal forms. From these notions, we also propose a confrontation with the reading made by Agamben.

* Doctor en Filosofía por la Universidad de Friburgo (Suiza), investigador principal del CONICET (INEO), miembro del Centro de Investigaciones Filosóficas (Buenos Aires) y profesor titular ordinario de Historia de la filosofía contemporánea (UNSAM). Sus publicaciones se ocupan de la filosofía contemporánea, particularmente francesa e italiana. Entre sus libros, cabe destacar Pensar a Foucault (1995), Giorgio Agamben. Una arqueología de la potencia (2008), Lecturas foucaulteanas. Una historia conceptual de la biopolitica (2011), Introducción a Foucault (2014) y Diccionario Foucault (2018). Dirección electrónica: edgardomanuelcastro@gmail.com 
Key words: Marginalistic integration, political utilitarianism, Biopolitics, Tanatopolitics, juridical forms

\section{Soberanía y biopolítica}

La cuestión del derecho en las investigaciones de Michel Foucault ha dado lugar a un intenso debate en la literatura secundaria sobre al autor, sobre todo en lengua inglesa y en el marco de los denominados Governamental Studies. Sin entrar en mayores detalles al respecto, nos encontramos, por un lado, con quienes sostienen el carácter antijurídico de los trabajos foucaultianos o, según una célebre expresión de Alan Hunt, ${ }^{1}$ con la expulsión del derecho de la Modernidad. El propio Foucault, sosteniendo que a partir de finales del siglo XVIII hemos ingresado en una fase de "regresión de lo jurídico", ha motivado estas posiciones. ${ }^{2}$ Pero, por otro, también nos encontramos con quienes han sostenido, como Golder y Fitzpatrick, ${ }^{3}$ que, en los trabajos del pensador francés, hallamos una concepción del derecho plenamente adecuada con el desarrollo de la Modernidad, desde el momento en que lo concibe como una forma vacía de polivalente funcionalidad. Entre un extremo y el otro, tampoco faltan las posiciones intermedias, como, por ejemplo, las de Nikolas Rose y Mariana Valverde, ${ }^{4}$ cuyo interés se focaliza en el modo en el que las formas del derecho se entrelazan con las del ejercicio del moderno biopoder, descrito por Foucault en el apartado final de La Volonté de savoiry en sus cursos en el Collège de France de la década de 1970.5

Aunque sin remitirse al debate antes mencionado, la relación entre derecho y Modernidad, particularmente respecto de Foucault, constituye el eje de varios de los trabajos de Giorgio Agamben que conforman la serie Homo

\footnotetext{
${ }^{1}$ Hunt, Alan, "Foucault's Expulsion of Law. Toward a Retrieval”, Law \& Social Inquiry, Vol. 17, No 1 (1992), p. 2.

${ }^{2}$ Foucault, Michel, La Volonté de savoir. Histoire de la sexualité 1, París, Gallimard, 1976, p. 190.

${ }^{3}$ Golder, Ben y Fitzpatrick, Peter, Foucault's law, Nueva York, Routledge, 2009, p. 85.

4 Véase Rose, Nikolas y Valverde, Mariana, “Governed by Law?”, Social and Legal Studies 7, 4 (1998), pp. 541-551.

5 Acerca de este debate, Golder y Fitzpatrick nos ofrecen un panorama general en el primer capítulo de su Foucault's law, pp. 11-52.
} 
sacer. Sobre todo del primero de ellos y el que más influencia ha ejercido sobre la relectura de los escritos foucaultianos y la recepción de sus trabajos editados póstumamente. En efecto, en Homo sacer. Il potere sovrano e la nuda vita, luego de una serie de consideraciones críticas sobre el pensador francés, refiriéndose a la noción de biopolítica, Giorgio Agamben sostiene:

La tesis foucaultiana deberá, entonces, ser corregida o, al menos, integrada, en el sentido de que lo que caracteriza a la política moderna no es tanto la inclusión de la zoe en la polis, en sí misma antiquísima, ni simplemente el hecho de que la vida como tal se convierta en el objeto eminente de los cálculos y las previsiones estatales [la biopolítica]. Lo decisivo es, más bien, que, junto al proceso por el cual la excepción se convierte en la regla, el espacio de la vida desnuda, situada en el origen al margen del ordenamiento, progresivamente termina coincidiendo con el espacio político $(\ldots){ }^{6}$

De este modo, Agamben no sólo retrotrae la cronología foucaultiana de la inclusión de la vida biológica en los mecanismos de la política hasta la misma Antigüedad, sino que, sobre todo, la explica sirviéndose del concepto de excepción soberana, estableciendo una relación de causa a efecto. Porque en la Modernidad la excepción se ha convertido en regla, la vida desnuda, la zoe, coincide con el espacio político. Según la expresión del propio autor, "la prestación fundamental del poder soberano es la producción de vida desnuda", 7 es decir, una vida expuesta a la muerte violenta, que no está protegida ni por las leyes de los hombres ni de los dioses y, por lo tanto, de la que se puede disponer sin cometer homicidio y sin necesidad de celebrar sacrificios. Desnuda, según los términos italianos utilizados por el autor, porque resulta uccidibile e insacrificabile. ${ }^{8}$ Esta vida es, precisamente, la del homo sacer. ${ }^{9}$

${ }^{6}$ Agamben, Giorgio, Homo sacer. Il potere sovrano e la nuda vita, Turín, Einaudi, 1995, p. 12. Excepto que indiquemos lo contrario, las traducciones son nuestras.

${ }^{7}$ Agamben, Giorgio, Homo sacer..., p. 202.

${ }^{8}$ Agamben, Giorgio, Homo sacer..., p. 91.

${ }^{9}$ Vale la pena señalar que, si bien es con la publicación del primer volumen de la serie que el bomo sacer adquiere el relieve que ha tenido en la corriente biopolítica -sirviendo de título para este primer volumen y, luego, para toda la serie-, su figura había sido introducida por el autor más de una década antes, en Il linguaggio e la morte, a propósito 
Como vemos, Agamben borra la distinción foucaultiana entre soberanía y biopolítica en favor de una concepción jurídica de esta última: la soberanía es biopolítica y la biopolítica, a su vez, ejercicio del poder soberano. Y, como consecuencia de ello, mientras para Foucault, no se trata de "hacer jugar la muerte en el campo de la soberanía, sino de distribuir lo viviente en un dominio de valor y de utilidad", 10 en el centro de la biopolítica agambeniana nos encontramos con la completa desprotección de la vida.

Tocamos aquí un punto que divide las aguas entre ambos pensadores. El propio Agamben es más que explícito al respecto. Según sostiene, el abandono de los modelos jurídico-institucionales le habría impedido a Foucault vincular sus investigaciones con los "lugares por excelencia de la biopolítica", los campos de concentración y los Estados totalitarios. ${ }^{11}$

Acerca de esta interpretación del pensamiento de Foucault por parte de Giorgio Agamben, son necesarias dos observaciones. El interés de Agamben por los trabajos de Foucault en la serie Homo sacer gira en torno a tres cuestiones fundamentales: la noción de biopolítica, a la que acabamos de referirnos, la idea de una genealogía teológica de la economía y del gobierno, abordada en Il Regno e la Gloria (volumen II, 2 de la serie, aparecido en el 2007) y la noción de archivo en relación con la de testimonio en Quel che resta di Auschwitz (volumen III de la serie, publicado en 1998). ${ }^{12}$ Si dejamos de lado esta última remisión, que aquí

de la discusión sobre la ambigüedad de lo sagrado (Agamben, Giorgio, Il linguaggio e la morte, Turín, Einaudi, 1982, p. 132). En este escrito, producto de un seminario dictado durante los años 1979-1980, Agamben remite frecuentemente a la obra de Émile Benveniste a propósito de la noción lingüística de shifters; pero no remite a su Vocabulaire des instituions indo-européenes, donde Benveniste, precisamente sobre la ambigüedad de lo sagrado en el universo indoeuropeo, alude a la figura del homo sacer (Benveniste, Émile, Le Vocabulaire des institutions indoeuropéenes, París, Minuit, 1969, tomo 2, p. 189).

${ }^{10}$ Foucault, Michel, La Volonté de savoir, p. 189.

${ }^{11}$ Ver: Agamben, Giorgio, Homo sacer..., pp. 7-8.

${ }^{12}$ La primera referencia de Agamben a los trabajos de Michel Foucault se remonta a 1978, a su Infanzia e storia (Turín, Einaudi, 1978, p. XI), a propósito de la idea de un ser bruto del lenguaje. Ésta es la única previa a las de Homo sacer I. Además de las remisiones al pensamiento foucaultiano en la serie Homo sacer, deben tenerse en cuenta, a la hora de establecer un balance intelectual entre ambos filósofos, la interpretación que nos ofrece Agamben de la noción de dispositivo (Agamben, Giorgio, Che cos'è un dispositivo?, Roma, Nottetempo, 2006) y su interés metodológico por la noción de arqueología 
no nos interesa, no puede dejar de subrayarse que, en Il Regno e la Gloria, Agamben, cuando reformula su concepción de la máquina política de Occidente, se refiere a los análisis foucaultianos sobre la gubernamentalidad en términos mucho menos críticos de los utilizados en el primer volumen de la serie, aunque elaborando una genealogía del gobierno y del liberalismo en una perspectiva teológica (los tratados sobre la Trinidad y sobre el gobierno divino del mundo) ausente en el pensador francés. Más allá de este posterior acercamiento a la obra de Foucault en Il Regno e la Gloria, Agamben no ha variado su postura respecto de la noción de biopolítica.

Segunda observación. En Homo sacer. Il potere sovrano e la nuda vita (en adelante Homo sacer I) Agamben remite, entrecomillada, aunque ahora para coincidir plenamente con él, a una cita de Foucault donde, refiriéndose a la transformación del Estado territorial al Estado poblacional, se sostiene:

Como consecuencia de ello se produce una especie de animalización del hombre llevada a cabo a través de las más sofisticadas técnicas políticas. Aparecen, entonces, en la historia tanto el desarrollo de la posibilidad de las ciencias humanas y sociales como la simultánea posibilidad de proteger la vida y de autorizar el holocausto. ${ }^{13}$

Esta cita, en la que en gran medida se apoya la lectura agambeniana de Foucault, aparece, como señalamos, entre comillas, pero sin ninguna referencia precisa en las sucesivas ediciones de la obra publicadas en la editorial Einaudi. Por otro lado, tampoco aparece en ninguno de los textos de Foucault publicados hasta la fecha en lengua original. Los términos "animalización" y "holocausto", además, son muy poco frecuentes en el vocabulario foucaultiano. ${ }^{14}$ La cita en cuestión proviene, en realidad, de una referencia que

(Agamben, Giorgio, Signatura rerum. Sul metodo, Turín, Bollati Boringhieri, 2008, pp. 7-8, 83 y ss).

13 Agamben, Giorgio, Homo sacer..., p. 5.

14 En los textos de Michel Foucault publicados hasta la fecha, el término "animalización" aparece, en efecto, apenas una única vez, en relación con una interpretación del deseo en Galeno (Foucault, Le Souci de soi. Histoire de la sexualité 3, Paris, Gallimard, 1984, p. 160). Respecto de "holocausto", aparece apenas dos veces (Foucault, Dits et écrits, París, Gallimard, 1994, t. 3, p. 278, y Foucault, La Volonté de savoir. Histoire de la sexualité 1, París, Gallimard, 1976, p. 179). 
se encuentra en la obra de Hubert Dreyfus y Paul Rabinow, Michel Foucault, Beyond Structuralism and Hermeneutics, quienes la atribuyen a Foucault como parte de las expresiones vertidas en las conferencias dictadas en la Universidad de Stanford en $1979 . .^{15}$ En la reciente edición integral de la serie Homo sacer, publicada por la editorial Quodlibet, que contiene en un solo volumen todos los tomos que la componen, ha sido agregada esta referencia. ${ }^{16}$

Ahora bien, entre el referido debate acerca de la cuestión del derecho en el pensamiento foucaultiano y la posición de Giorgio Agamben pueden marcarse puntos de coincidencia y de divergencia que le confieren, sin duda, una particular fisonomía a la posición del pensador italiano. En cuanto a las coincidencias, para restringirnos un punto nodal, señalemos que la denuncia del antijuridicismo foucaultiano por parte de Agamben se superpone con la concepción de la ley como forma vacía, con la que Golder y Fiztpatrick pretenden superar esta tesis. ${ }^{17}$ Respecto de las divergencias, también deteniéndonos en un punto particularmente significativo, la posición de Agamben va mucho más allá de la cuestión de la subordinación del derecho a las formas modernas del poder normalizador, las disciplinas y la biopolítica de la población, o de las posibilidades de complementariedad entre ellos, para afirmar la necesidad de identificar por completo soberanía y biopolítica. ${ }^{18}$

15 Dreyfus, Hubert y Rabinow, Paul, Michel Foucault: Beyond structuralism and hermeneutics, Brighton, Harvester Press, 1982, p. 138.

16 Ver: Agamben, Giorgio, Homo sacer. Edizione integrale, Macerata, Quodlibet, 2018, p. 19. Excepto aquí, en todos los demás casos citamos la primera edición de esta obra, de 1995.

17 Golder y Fitzpatrick remiten, de hecho, a la posición agambeniana (Golder, Ben y Fitzpatrick, Peter, Foucault's law, p. 83).

18 A propósito de esta identificación agambeniana, Tom Frost ha hablado, precisamente, de una legalización (legalization) de Foucault. Ver: Frost, Tom, "Agamben's Sovereign Legalization of Foucault", Oxford Journal of Legal Studies, Vol. 30, No 3 (2010), pp. 545-577. Sin poder entrar ahora en detalles acerca de la calificación de Tom Frost, dado que trasciende los objetivos de nuestro trabajo, en la medida en que, para Agamben, la vigencia de la ley coincide con sus suspensión, vale la pena preguntarse en qué sentido y con qué alcances se puede hablar de una legalización de Foucault por parte de Agamben, y, al límite, también, por la misma razón, si la acusación de un abandono de lo jurídico no puede, al menos cierto sentido, ser adecuada para la propia posición agambeniana. 
No es de nuestro interés discutir aquí las diferencias entra la noción foucaultiana de biopolítica, que no es de matriz jurídica ni necesariamente negativa, y la concepción agambeniana, que, precisamente, se define en los términos exactamente contrarios, como excepción soberana y exposición (desnudez) de la vida a la muerte violenta; sino, más bien, tomando como contrapunto las objeciones agambenianas, ocuparnos del modo en el que Michel Foucault aborda la cuestión del derecho y su relación con la vida en el pasaje del Estado territorial al Estado poblacional o, en otros términos, en la época de la razón de Estado y de la formación de la racionalidad política liberal.

Con este objetivo, retomaremos, primero, algunos de los elementos que definen la posición de Giorgio Agamben en Homo sacer I, en particular los que conciernen a las nociones de excepción y de culpa, es decir, por un lado, el mecanismo con el cual Agamben concibe la relación del derecho con la vida y, por otro, inversamente, de la vida con el derecho. Luego, en segundo lugar, intentaremos mostrar cómo se presenta esta doble relación, del derecho con la vida y de la vida con el derecho, en lo que Foucault describe como el ideal policial de la razón de Estado. Nos encontraremos aquí con dos nociones que ocupan, en el pensamiento foucaultiano, el lugar de la excepción: la noción de integración marginalista y la de golpe de Estado permanente. Seguidamente nos ocuparemos de la reformulación de estos conceptos a partir del advenimiento de la racionalidad política liberal, a través de las nociones de utilitarismo político y de heterogeneidad entre las formas de limitación interna de la acción gubernamental y las formas de la ley como expresión de la voluntad soberana.

Antes de proceder en esta dirección, debemos tener en cuenta, en primer lugar, que la problemática que abordamos aquí no agota, de ninguna manera, la cuestión del derecho en el pensamiento de Michel Foucault y, por lo tanto, tampoco las posibles y necesarias respuestas a la denominada tesis expulsionista. En este sentido, otros dos grandes capítulos deberían complementar el camino que aquí nos proponemos emprender. Por un lado, el que aborde la función metodológica que ha desempeñado, en la genealogía foucaultiana de la Modernidad, la historia del derecho a partir de su relación con la historia de la verdad, y que constituye el eje de sus primeros cursos en el Collège de France, de los que las conferencias en Río de Janeiro de 1973, “La vérité et les formes juridiques", ${ }^{19}$ son una versión resumida y articulada. Por

${ }^{19}$ Ver: Foucault, Michel, Dits et écrits, París, Gallimard, 1994, t. 2, pp. 139 y ss. 
otro lado, también deberían abordarse las nociones foucaultianas de derecho de los gobernados y de nuevo derecho. ${ }^{20}$ Ambos capítulos, de todos modos, trascienden el objetivo de nuestro trabajo.

En segundo lugar, también es necesario señalar que la finalidad de la confrontación que nos proponemos con la tesis agambeniana sobre el antijuridicismo de Michel Foucault no consiste tanto en ofrecer una respuesta a una objeción formulada por el filósofo italiano en el primer volumen de la serie Homo sacer, del que se cumplen ya veinticinco años de su publicación; sino, sobre todo, abordar una problemática, la de las relaciones entre derecho y vida, cuya actualidad trasciende la cronología de las publicaciones y cuya vigencia continúa siendo de relevancia en la interpretaciones del pensamiento foucaultiano.

\section{La captura y la culpa}

Una cita de Friedrich Carl von Savigny sirve de exergo a Homo sacer. Il potere sovrano e la nuda vita: el derecho no tiene en sí mismo ninguna existencia; su esencia consiste, más bien, en la vida misma de los hombres. El entero capítulo primero de la obra puede considerarse como la justificación de esta referencia y la explicitación de su significado para el autor. Se trata, precisamente, del capítulo donde Agamben, enfatizando la dimensión paradójica, expone su concepción de la excepción soberana o, si preferimos, de la topología de la excepción. Según sostiene, no se trata:

ni [de] un concepto exclusivamente político, ni [de] una categoría exclusivamente jurídica, ni [de] una potencia externa al derecho (Schmitt), ni [de] la norma suprema del ordenamiento jurídico (Kelsen). Ella [la soberanía] es la estructura con la que el derecho se refiere a la vida y la incluye en sí misma a través de la propia suspensión. ${ }^{21}$

Agamben pone el acento, como puede verse, en la manera en que, por la excepción soberana, el derecho "captura" la vida. ${ }^{22}$ Respecto de la noción de captura, precisamente en relación con la lectura agambeniana de Foucault, es

\footnotetext{
${ }^{20}$ Ver: ibid., t. 3, p. 361.

${ }^{21}$ Agamben, Giorgio, Homo sacer..., p. 34.

${ }^{22}$ Ibid., p. 32.
} 
necesario subrayar que ella trasciende el ámbito de la reflexión sobre los conceptos jurídicos para convertirse en una categoría ontológica del autor. En efecto, una década más tarde de la aparición de Homo sacer I, retomando el gesto de Gilles Deleuze -aunque sin remitirse explícitamente a él-, también Agamben dedica una conferencia a la noción foucaultiana de dispositivo. Con la intención de ofrecer una lectura ontológica de este concepto, por un lado, lleva a cabo una genealogía de la noción de dispositivo en el marco del pensamiento foucaultiano, vinculándola, a través de la figura de Jean Hyppolite, con la idea hegeliana de positividad. Por otro, extendiendo el campo de análisis, esboza una genealogía teológica del dispositivo a partir de la noción de oikonomia, que los Padres latinos de la Iglesia tradujeron, precisamente, por dispositio. Al final de este recorrido, Agamben propone la división de todo lo existente en dos categorías ontológicas fundamentales: las substancias y los dispositivos que las capturan. ${ }^{23}$ Desde este punto de vista, la soberanía, en particular, y el derecho, en general, serían, entonces, casos dentro de una ontología en la que todos los dispositivos se definen por su capacidad de captura.

Esta captura es la que le confiere a la soberanía su carácter paradójico, estableciendo entre derecho y vida una relación de exclusión inclusiva. ${ }^{24}$ Si ser soberano, según la posición schmittiana, es decidir acerca del estado de excepción, es decir, sobre la suspensión del ordenamiento jurídico; por un lado, entonces, nos encontramos con aquello que queda fuera de ese ordenamiento, la vida. Pero, por otro, esa exclusión no podría ser definida como tal si lo excluido no estuviese, de algún modo, en relación con ese ordenamiento jurídico del que, precisamente, está excluido.

En esta relación de exclusión inclusiva que define la topología de la excepción soberana, no se trata de que, respecto de la vida, el derecho establezca una sanción, sino de que la vida está en culpa. Y, en este sentido, Agamben precisa: "La culpa no se refiere a la transgresión, es decir, a la determinación de lo lícito y lo ilícito, sino a la pura vigencia de la ley, a su simple referirse a algo". ${ }^{25}$ Como en la expresión alemana utilizada por Walter Benjamin "bloßes Leben", el adjetivo "bloßes", traducido al italiano por "nuda" y al español por "desnuda",

\footnotetext{
${ }^{23}$ Ver: Agamben, Giorgio, Che cos'è un dispositivo?, pp. 21-22.

${ }^{24}$ Agamben, Giorgio, Homo sacer..., p. 11.

${ }^{25}$ Ibid., p. 32.
} 
pero que también hubiese podido ser traducido por "simple", le da a la noción de vida toda su problematicidad; ${ }^{26}$ en el párrafo apenas citado, lo mismo ocurre con el "simple" que, en este caso, adverbia al "referirse". Se trata de una referencia sin significado, sin contenido, un "simple" referirse respecto del cual la vida está siempre en culpa.

Al igual que el mecanismo por el cual la vida es objeto de una captura, también la noción de culpa constituye uno de los temas recurrentes en el pensamiento agambeniano, más allá de los desarrollos de Homo sacer I, explicitando, de este modo, la densidad conceptual de este primer volumen de la serie. En un trabajo reciente, Karman. Breve tratatto sull'arione, la colpa e il gesto (2017), Agamben sostiene acerca de los conceptos de causa y de culpa que "no son conceptos jurídicos en sentido estricto, sino que marcan -como vimos- el punto en el que un determinado acto o hecho entra en la esfera del derecho. (...) En este sentido, culpa es sinónimo de noxa, un término cuya etimología remite a la esfera oscura de la muerte violenta (nex)."27

Respecto de la posición foucaultiana, como veremos, también es necesario tener presente que, en la concepción agambeniana, la relación entre captura y culpa responde a la necesidad del orden o, mejor, a la imposibilidad de aplicar la ley al caos. El ordenamiento jurídico presupone, insiste Agamben, una "estructuración normal de las relaciones de vida", "la norma [jurídica] tiene necesidad de una situación media homogénea". 28

Por último, señalemos que, para Agamben, puesto que aquello que está en juego en la relación de exclusión inclusiva, producto de la decisión soberana, es el modo en que el derecho captura la vida -es decir, el estado de excepción y no el derecho penal-, una investigación sobre los campos de concentración y exterminio no podría haberse desarrollado a partir del "camino abierto por los trabajos de Foucault, desde la Histoire de la folie a Surveiller et punir" ${ }^{29}$ En los campos, en efecto, no se trata propiamente de la mera reclusión o del encierro,

\footnotetext{
${ }^{26}$ Sobre esta problematicidad, ver: Moran, Brendan y Salzani, Carlo, Towards the critique of violence: Walter Benjamin and Giorgio Agamben, Londres/Nueva York, Bloomsbury, 2015, pp. 109-123.

27 Agamben, Giorgio, Karman: Breve trattato sull'azione, la colpa e il gesto, Turín, Bollati Boringhieri, 2017, p. 16.

28 Agamben, Giorgio, Homo sacer..., pp. 19-20.

${ }^{29}$ Ver: ibid., p. 24.
} 
de acuerdo con lo permitido y lo prohibido; sino de la exclusión de la vida en relación con la "simple" vigencia de la ley. Por ello, sostiene, no es la cárcel sino el campo el paradigma de la Modernidad. ${ }^{30}$

Sin entrar en mayores detalles al respecto, vale la pena llamar la atención sobre un pasaje de la Histoire de la folie que parece haber pasado inadvertido al autor de Homo sacer. Aquí, en su interpretación de Sade, Foucault se aproxima fuertemente a la concepción agambeniana de la soberanía. Se trata de las páginas finales de esta obra donde, luego de haber descrito la formación del homo psychologicus, se detiene en los espacios pictóricos y literarios, Goya y Sade, en los que, a pesar de haber ser sido reducida a silencio durante la Época clásica (siglos XVII y XVIII), la locura hace todavía escuchar su voz. La intención del autor, en estas páginas, no es referirse directamente a la soberanía estatal, sino a la subjetividad de los personajes de la Société des amis du crime; pero, en la medida en que la filosofía de Rousseau aparece como contrapunto, estas páginas no dejan de tener una connotación política. Refiriéndose a las acciones de los miembros de dicha sociedad, Foucault sostiene:

la disposición incontrolada de uno sobre otro, el ejercicio desmesurado de violencia, la aplicación sin límite del derecho de muerte -toda esta sociedad, cuyo único vínculo es el rechazo de todo vínculo, aparece como el abandono de la naturaleza-, la única cohesión que se les exige a los individuos del grupo no tiene como sentido proteger una existencia natural, sino el libre ejercicio de la soberanía sobre y contra la naturaleza. ${ }^{31}$

Esta desatención de la lectura agambeniana de la Histoire de la folie llama particularmente la atención, porque, para el autor de Homo sacer, el panfleto de Laphilosophie dans le boudoir leído por Dolmancé, constituye "el primero y quizás más radical manifiesto biopolítico de la Modernidad", puesto que "la misma vida fisiológica de los cuerpos se presenta como elemento político puro". 32

\section{Integración marginalista y utilitarismo político}

\footnotetext{
30 Ver: ibid., pp. 24, 189.

${ }^{31}$ Foucault, Michel, Histoire de la folie à l'âge classique, París, Gallimard, 1999, p. 658.

32 Agamben, Giorgio, Homo sacer..., p. 149.
} 
En los escritos foucaultianos publicados hasta la fecha, la expresión "estado de excepción" aparece apenas dos veces. ${ }^{33}$ Mucho más frecuente es la expresión "estado de guerra", utilizada sobre todo a propósito de la tesis hobbesiana de esa lucha de todos contra todos que precede al surgimiento del Estado. ${ }^{34} \mathrm{Al}$ igual que respecto del derecho, Foucault no ha elaborado ninguna teoría acerca de la excepción soberana. Esto no significa que el ejercicio de la soberanía y la relación entre el derecho y la vida hayan quedado afuera de su problematización de la política moderna. De hecho, esta última ha sido uno de los temas privilegiados por los especialistas, particularmente en el debate al que nos hemos referido al inicio; pues involucra, en los trabajos de nuestro autor, la relación entre la ley en su sentido jurídico y el proceso moderno de normalización, al que ha dedicado varios de sus trabajos. ${ }^{35}$ Pero esta problemática, a pesar de la importancia que tiene en sus escritos, en la literatura filosófica al menos, no aparece suficientemente vinculada con la noción de policía, que ha quedado de algún modo opacada por otras o en segundo plano respecto de ellas, como las de disciplina y biopolítica. ${ }^{36}$ A nuestro modo de ver, por las razones que se mostrarán seguidamente, tanto un contrapunto con la

${ }^{33}$ Foucault, Michel, Dits et écrits, t. 4, p. 340 y Foucault, Michel, Naissance de la biopolitique Cours au Collège de France. 1978-1979, París, Gallimard-Seuil, 2004, p. 160, nota 36. En este último caso en una nota del editor.

34 Por ejemplo, Foucault, Michel, "Il faut defendre la société". Cours au collège de France. 1976, París, Gallimard-Seuil, 1997, pp. 79-80; La société punitive. Cours au Collège de France. $1972-$ 1973, París, EHESS-Seuil-Gallimard, 2013, pp. 26-27.

${ }^{35}$ Foucault, Michel, Surveiller et punir, París, Gallimard, 1975, p. 185.

36 Acerca de esta desatención en el campo de la literatura filosófica por la noción de policía, téngase presente, por ejemplo, que ningún artículo específico le está dedicado en el Dictionnaire Foucault (de Revel, Judith, París, Ellipses, 2008), en el Foucault Lexikon (de Ruoff, Michael, Padeborn, Wilhem Fink, 2009) o en el Cambridge Foucault Lexicon (de Lawlor, Leonard y Nale, John, Nueva York, Cambridge University Press, 2014). Al respecto, ver: Castro, Edgardo, "La noción de policía en los trabajos de Michel Foucault: objeto, límites, antinomias", Anuario colombiano de historia social y de la cultura, 46, 2 (2019), p. 188. En el campo de la historia, en cambio, la noción foucaultiana de policía ha recibido un tratamiento significativamente más amplio (ver: Denis, Vincent, "L'histoire de la police après Foucault. Un parcours historien", Revue d'bistoire moderne et contemporaine, t. 60e, $\mathrm{N}^{\mathrm{0}} 4 / 4$ bis (2013), pp. 139-155). En este campo, ocupa un lugar destacado el trabajo de Paolo Napoli, La naissance de la police moderne. Pouvoir, normes, société (París, La Découverte, 2003). 
interpretación agambeniana como la comprensión de esta problemática en sí misma deben partir precisamente de aquí.

En este sentido, vale la pena subrayar que cuando Agamben aborda la noción de policía, remitiéndose explícitamente a Foucault, como en Homo sacer I, lo hace para hablar de la distinción que se establece, en el campo de la denominada ciencia de la policía del siglo XVIII (Polizeimissenschaft), entre política (la relación entre los Estados) y policía (la política interior), de la dimensión eugenésica de esta última y de la tendencia contemporánea a borrar esa distinción cuando se llevan a cabo acciones de policía en territorio extranjero. ${ }^{37}$ Pero en ninguno de estos casos, a propósito de la noción moderna de policía, Agamben se detiene en la relación entre el derecho y la normalización de la vida.

La cronología de la publicación de los cursos de Michel Foucault en el Collège de France explica, en gran medida, esta situación. En Sécurité, territoire, population, en efecto, sobre la relación entre ley y orden, Foucault habla de la policía como de un "golpe de Estado permanente", 38 es decir, con un concepto que se superpone con el de estado de excepción o, al menos, se aproxima en gran medida. Pero, en este caso, se trata de un curso publicado recién en el 2004 y, por lo tanto, casi diez años más tarde que Homo sacer I. Aunque también es necesario notar que parte de este material circuló, en vida del autor, a través de artículos e intervenciones, reunidos posteriormente en la compilación titulada Dits et écrits de 1994. Entre estos textos y en relación con el tema que aquí nos interesa, no pueden dejar de mencionarse "Omnes et singulatim. Towards a Criticism of Political Reason", 39 una conferencia de 1979, y "The Political Technology of Individuals", 40 un curso de 1982, a los que sí remite Agamben en el primer volumen de la serie Homo sacer. ${ }^{41}$

Desde un punto de vista conceptual, sin embargo, más allá de la historia de la recepción, los análisis de Michel Foucault en torno al nacimiento de la policía moderna constituyen, como señalamos, un requisito indispensable para

\footnotetext{
37 Ver: Agamben, Giorgio, Homo sacer..., pp. 161 y ss..

38 Foucault, Michel, Sécurité, territoire, population. Cours au Collège de France. 1977-1978, París, Gallimard-Seuil, 2004, p. 347.

${ }^{39}$ Foucault, Michel, Dits et écrits, t. 4, pp. 134-161.

${ }^{40}$ Ibid., t. 4, pp. 813-828.

41 Agamben, Giorgio, Homo sacer..., pp. 7-8, 161.
} 
comprender la relación entre derecho y vida en su ontología histórica de la Modernidad.

En este sentido, en particular a partir del cuantioso material publicado póstumamente, es posible hablar de lo que bien puede definirse como un tratado foucaultiano sobre la policía moderna, compuesto por cinco lecciones fundamentales: las dos últimas clases del curso Sécurité, territoire, population, la primera del curso siguiente, Naissance de la biopolitique, la titulada "The Political Technology of Individuals", del seminario dictado en Vermont en 1982, y la mencionada conferencia “Omnes et singulatim" de las Tanner Lectures de 1979. En este tratado, Foucault se ocupa del objeto, los límites y las antinomias de la policía, tomando como autores de referencia, sobre todo, la obra de Johann von Justi y Nicolas Delamare. ${ }^{42}$

Debido a nuestro objetivo, nos interesa señalar que, en este tratado, a propósito de la relación entre el Estado moderno y la vida de los individuos, Foucault habla ni más ni menos que de un "nuevo sistema antropológico". ${ }^{43}$ En cuanto al Estado, el énfasis de nuestro autor no está puesto en lo que, para Agamben, define el ejercicio de la soberanía, es decir, la decisión acerca de la excepción; sino en la racionalidad de las formas de intervención para conducir la vida de los individuos y de la población. Y respecto de la vida, también aquí a diferencia de Agamben, no está en cuestión solo la simple vida natural, la vida biológica, sino también la vida social o, según el lenguaje de los tratadistas de la época, la felicidad de los hombres. Pues se trata de un sistema que va, según la expresión de Foucault, "del vivir al más que vivir". ${ }^{44}$ Entre el Estado, por un lado, y la vida, por el otro, se ubica precisamente la policía; entendiendo por policía, precisamente, esas formas de intervención del Estado, racionales y calculadas, sobre el vivir y el más que vivir de los ciudadanos.

La felicidad de los hombres, según los tratadistas analizados por Foucault, constituye el objetivo del poder de policía del Estado; a pesar de ello, según estos mismos tratadistas, el Estado puede también exigir la muerte de sus ciudadanos. ${ }^{45}$ Existe, en este sentido, y Foucault insiste en este aspecto, una coexistencia característica en la racionalidad política moderna -no sólo a

\footnotetext{
${ }^{42}$ Ver: Castro, Edgardo, La noción de policía..., p. 192.

${ }^{43}$ Foucault, Michel, Sécurité, territoire, population, p. 334.

${ }^{44}$ Ibid., p. 333.

45 Ver: Foucault, Michel, Dits et écrits, t. 4, pp. 819-820.
} 
propósito del ideal policial de la razón de Estado- entre los fenómenos de destrucción masiva y la puesta en funcionamiento de las instituciones de protección de la vida. ${ }^{46}$ Pero, más allá de esta coexistencia, es necesario insistir en que la intervención del Estado sobre la vida de los individuos y de la población, su poder de policía, en los términos en que lo hemos definido más arriba, no reviste necesariamente un carácter negativo. El apartado final de $L a$ Volonté de savoir es, en este sentido, por demás de elocuente. Foucault, en efecto, habla de mantenimiento, desarrollo y mejora de la vida ${ }^{47}$ o, según otra formulación a la que ya nos hemos remitido no se trata de "hacer jugar la muerte en el campo de la soberanía, sino de distribuir lo viviente en un dominio de valor y de utilidad". 48

Foucault define el funcionamiento de este nuevo sistema antropológico, que toma forma con el surgimiento de la policía en el sentido moderno, en términos de "integración marginalista" 49 y de "golpe de Estado permanente". ${ }^{50}$ $\mathrm{Al}$ respecto sostiene:

En esta nueva racionalidad política [la del Estado moderno], ella [la integración marginalista] se alcanza mediante una técnica bien particular que se llamaba, por ese entonces, la policía. (...) Frecuentemente, cuando se analiza la función del Estado en nuestra sociedad, o uno se concentra en las instituciones -el ejército, la función pública, la burocracia o instancia semejantes- o se analizan las teorías o las ideologías elaboradas con la finalidad de justificar o de legitimar la existencia del Estado.

Lo que yo busco, al contrario, son las técnicas, las prácticas que dan una forma concreta a esta nueva racionalidad política y este nuevo tipo de relación entre la entidad social y el individuo. ${ }^{51}$

Si bien la expresión "integración marginalista", en el estado actual de la publicación de sus escritos, aparece sólo aquí, en la mencionada conferencia "The Political Technology of Individuals", en la medida en que es utilizada,

\footnotetext{
${ }^{46}$ Foucault, Michel, Dits et écrits, t. 4, p. 815; La Volonté de savoir, p. 180.

${ }^{47}$ Foucault, Michel, La Volonté de savoir, pp. 176, 181.

48 Ibid., p. 189.

${ }^{49}$ Foucault, Michel, Dits et écrits, t. 4, p. 820.

${ }^{50}$ Foucault, Michel, Naissance de la biopolitique, p. 74.

51 Foucault, Michel, Dits et écrits, t. 4, p. 820.
} 
como acabamos de señalar, para definir ese nuevo sistema antropológico, su importancia no puede ser soslayada. En primer lugar, por el carácter conceptualmente problemático o, si se prefiere, anfibológico de la expresión, que reúne la noción de integración con la de marginalidad, haciendo de la utilidad el nexo entre ambas y, de este modo, permitiendo explicar la característica contemporaneidad de las formas de protección y de destrucción de la vida en la Modernidad. Y, en segundo lugar, porque Foucault se sirve de este concepto político de utilidad, como veremos seguidamente, cuando habla del punto de anclaje de la racionalidad política liberal y, como ya vimos, también cuando describe la biopolítica como distribución de la vida, precisamente, en un campo de utilidad. De este modo, Foucault utiliza una noción proveniente del ámbito de la teoría económica, confiriéndole un sentido plenamente político. Pues se trata, según las palabras propias del autor, de un "marginalismo político", 52 donde el Estado se interesa por la vida de los individuos y se ocupa de ella sólo en la medida en que le resulta beneficiosa para aumentar su propia potencia y no la de la vida en sí.

Por esta razón, el Estado puede no sólo exigir la muerte de sus ciudadanos; independientemente del ejercicio del poder soberano de castigar con la muerte o declarar la guerra a los enemigos del Estado, puede también simplemente descartar hacia la muerte, "rejeter dans la mort", ${ }^{33}$ la vida de los individuos y de la población. En este caso, en el descartar hacia la muerte (rejeter dans la mort) de la biopolítica foucaultiana, nos encontramos, finalmente, con una vida que resulta ser más desnuda que la agambeniana nuda vita, puesto que no requiere ni estar en relación con ninguna forma jurídica ni de la exposición a una muerte violenta, sino que está en juego sólo su inutilidad.

Ahora bien, la policía, entendida como la intervención directa del soberano sobre la vida de los individuos, sin pasar por las instancias de la justicia, sus leyes y sus instituciones, es el instrumento y la posibilidad misma del marginalismo político en el marco de la razón de Estado. De la soberanía emanan, entonces, el poder de justicia y el poder de policía; pero este último no se subordina al anterior, se ejerce de manera directa y, por lo tanto, paralela. ${ }^{54}$ Por esta razón, Foucault habla de la policía como de un "golpe de

\footnotetext{
${ }^{52}$ Foucault, Michel, Dits et écrits, t. 4, p. 820.

${ }^{53}$ Foucault, Michel, La Volonté de savoir, p. 181.

${ }^{54}$ Foucault, Michel, Sécurité, territoire, population, p. 348.
} 
Estado permanente". En estas mismas páginas, aclara, sin embargo, que de ello no se sigue que la policía carezca de toda dimensión jurídica. Los tratadistas de la época, de hecho, hablan de un derecho de justicia y de un derecho de policía. Pero, en este último caso, no concierne a las disposiciones de carácter general, sino a las reglamentaciones que pueden alcanzar hasta los más mínimos detalles y, al menos teóricamente, tener un alcance ilimitado. 55

En la época de la Razón de Estado o del Estado de policía -Foucault utiliza de hecho ambas expresiones como sinónimos-, nos encontramos, entonces, con una configuración del ejercicio gubernamental de la soberanía estatal en el que, por un lado, se constituyen, según el análisis de Théories et institutions pénales, las instituciones estatales de justicia ${ }^{56} \mathrm{y}$, por otro, toman forma las técnicas de policía (órganos administrativos y de ejecución, reglamentaciones y tratadística) a través de las cuales el Estado se hace cargo de la vida biológica y social de los individuos y de la población, del vivir y del más que vivir.

Si bien, según los lineamiento generales de Sécurité, territoire, population, el proyecto de la razón de Estado se fragmenta dando lugar al ejercicio del gobierno según la economía, a la gestión biopolítica de la población, a un derecho público garante de las libertades individuales y a una policía reducida a su función represiva y de auxiliar de la justicia, ${ }^{57}$ la nueva racionalidad política surgida de esta fragmentación -esto es, la racionalidad política liberal-, seguirá funcionando en términos de utilidad y de oposición entre la ley como expresión de una voluntad soberana y el orden requerido de la vida social.

Para Foucault, aspecto sobre el que ha explícitamente insistido, el liberalismo, más que una doctrina económica, representa una concepción antropológica. Tomando distancia de las caracterizaciones habituales de la Modernidad en general y del liberalismo en particular, el objetivo del curso Naissance de la biopolitique no es concebirlo como el advenimiento o el fortalecimiento de la libertad y de la individualidad, sino de la seguridad y de la población o, para ser más precisos, de la articulación entre libertad y seguridad, entre individuo y población. Esta articulación o, según los términos utilizados

\footnotetext{
${ }^{55}$ Foucault, Michel, Naissance de la biopolitique, p. 9.

${ }^{56}$ Foucault, Michel, Théories et institutions pénales. Cours au Collège de France. 1971-1972, París, EHESS-Seuil-Gallimard, 2015, pp. 183-184.

57 Ver: Foucault, Michel, Sécurité, territoire, population, p. 362.
} 
por nuestro autor, el arbitraje ${ }^{58}$ entre estos elementos, estableciendo límites respecto de la acción de gobernar, define la especificidad del liberalismo. ${ }^{59}$

Foucault distingue, en este sentido, entre límites externos e internos al ejercicio gubernamental de la soberanía estatal. Los límites externos son los establecidos por el derecho público a través de los denominados derechos fundamentales, es decir, aquellos que, sea que estén fundados en una teoría del contrato o de la naturaleza humana, determinan la legitimidad o ilegitimidad del ejercicio del poder soberano. Ahora bien, la particularidad del liberalismo implica también la formulación de límites internos, esto es, a partir de la propia práctica gubernamental y de los saberes en los que se apoya y, a la vez, produce (como los de la economía o acerca de la población).

El mercado y la utilidad han servido, según Foucault, como puntos de anclaje, ${ }^{60}$ precisamente, de estas limitaciones internas de la práctica gubernamental. En un primer momento, en efecto, Foucault se detiene en el mercado como mecanismo de veridicción, ${ }^{61}$ es decir, como una instancia que sirve para establecer si se está gobernando bien o mal o, más precisamente, en exceso; alterando, en definitiva, el curso de las cosas y provocando el efecto contrario al deseado. Pero luego de haberse detenido en el mercado como forma de veridicción (concepto que introduce precisamente a propósito de esta cuestión y que guiará en gran parte sus investigaciones futuras), la utilidad o lo que denomina el utilitarismo político se convierte en el punto de anclaje general para establecer esas buscadas limitaciones internas a la acción de gobernar. Por eso, según la formulación foucaultiana, el éxito o el fracaso de la acción gubernamental va a substituir a la problemática de la legitimidad y de la ilegitimidad de la soberanía, y el utilitarismo, consecuentemente, va a penetrar el entero campo de la racionalidad política. ${ }^{62}$

Ahora bien, por un lado, Foucault no ve este proceso como la proyección de la filosofía utilitarista hacia el campo de la práctica gubernamental. Las relaciones entre el utilitarismo político y la filosofía

\footnotetext{
${ }^{58}$ Ibid., p. 67.

${ }^{59}$ Ver: Foucault, Michel, Naissance de la biopolitique, pp. 23 y ss.

${ }^{60}$ Ibid., p. 45.

${ }^{61}$ Ibid., p. 33.

${ }^{62}$ Ibid., pp. 18-19.
} 
utilitarista, sin que podamos detenernos en ello, en todo caso, son más complejas. $\mathrm{Al}$ respecto, sostiene:

(...) el utilitarismo [político, podríamos agregar] es algo completamente diferente de una filosofía, completamente diferente de una ideología. El utilitarismo es una tecnología de gobierno, así como el derecho público en la época de la razón de Estado era la forma de reflexión o, si se prefiere, la tecnología jurídica con la cual se trataba de limitar la línea de acción de la razón de Estado. ${ }^{63}$

Y, por otro, tampoco considera que esta invasión de la noción de utilidad en el campo de la racionalidad política significa que la cuestión el derecho haya sido dejada de lado. Ese mismo utilitarismo, para nuestro autor, también ha invadido el campo del derecho; de modo que, según sus propias expresiones, "la utilidad recubre cada vez más todos los problemas tradicionales del derecho". ${ }^{64}$

Precisamente, a propósito esta invasión de la problemática de la utilidad en la esfera del derecho, nos reencontramos con la segunda característica de la racionalidad política moderna, esto es, la oposición-complementación entre la ley y el orden, entre lo jurídico, retomando una caracterización introducida por François Ewald, entendido como la codificación de las relaciones verticales de soberanía, y la ley, entendida como la expresión de las relaciones horizontales de gubernamentalidad. ${ }^{65}$ A propósito de esta oposición-complementación en el marco de la racionalidad liberal, Foucault ya no habla de golpe de estado permanente, sino de heterogeneidad.

Heterogeneidad [sostiene] que no quiere decir contradicción, sino tensiones, fricciones, incompatibilidades mutuas, ajustes logrados o fallidos, combinaciones inestables, etc. Lo que quiere decir, también, tarea siempre retomada, porque nunca acabada, de establecer o una coincidencia o un régimen común. ${ }^{66}$

\footnotetext{
${ }^{63}$ Ibid., p. 42.

${ }^{64}$ Ibid., p. 45.

${ }^{65}$ Ewald, François, “Norms, Discipline, and the Law”, Representations, 30 (1990), p. 155.

${ }^{66}$ Foucault, Michel, Naissance de la biopolitique, p. 23.
} 
Y sostiene, además (dada su importancia en relación con las críticas al denominado antijuridicismo foucaultiano, nos permitimos otra cita del autor):

No es pues una desaparición del derecho lo que evocaba la última vez hablando de una autolimitación de la razón gubernamental, sino del problema planteado por la limitación jurídica que imponía fijar los problemas de la verdad al ejercicio del poder político. ${ }^{67}$

Ahora bien, si en la época de la razón de Estado nos encontrábamos, por un lado, con el principio de integración marginalista y con el de golpe de Estado permanente y, por otro, con el vivir y el más que vivir de los individuos y de la población; en la configuración propia de la racionalidad liberal, nos encontramos, por un lado, con el principio del utilitarismo político y con el de heterogeneidad entre la expresión jurídica de la voluntad soberana y la de las formas jurídicas de las limitaciones internas de la acción de gobernar y, por otro, en cuanto concierne a la vida, con la vida concebida en términos de riesgo, con el vivir en riesgo. ${ }^{68}$

Para Foucault éste es, propiamente hablando, el efecto del arbitraje liberal entre libertad y seguridad en términos de utilitarismo político. Los individuos y las poblaciones están, por eso, expuestos continuamente a la situación de riesgo o, al menos, a experimentar esta situación. No se trata aquí, es necesario subrayarlo, del riesgo de ser expuesto a la muerte violenta, como en la figura del homo sacer, de la aparición, según la fórmula utilizada por Foucault, de los cuatro jinetes del apocalipsis; sino de la emergencia y de la invasión del riesgo en todos los órdenes de la vida, de la biología a la psicología, de la salud a la enfermedad, de la economía a la sexualidad. Por eso, desde esta perspectiva, Foucault define el liberalismo como la cultura del riesgo. ${ }^{69}$

\section{Conclusión}

Del recorrido que hemos llevado a cabo podemos extraer algunas conclusiones, a nuestro juicio, relevantes. Según la posición de Giorgio Agamben, sobre la

${ }^{67}$ Ibid., p. 40.

${ }^{68}$ Acerca de la relevancia de la noción de riesgo en la concepción liberal de la vida social, ver: Ewald, François, "Norms, Discipline, and the Law", pp. 141 y ss.

${ }^{69}$ Foucault, Michel, Naissance de la biopolitique, p. 68. 
que nos hemos detenido al inicio de nuestro trabajo, más allá de la inclusión de la vida en los cálculos y mecanismos de la política, lo decisivo ha sido, por un lado, que la excepción se ha convertido en regla y que la vida desnuda, la prestación originaria del poder soberano, se superpone con el espacio político. Siempre según Agamben, la deficiencia de la posición foucaultiana -que, de acuerdo a sus expresiones, debe ser corregida o, al menos, completada- sería consecuencia de su abandono de los modelos jurídico-institucionales, que le habrían impedido vincular sus investigaciones con los lugares por excelencia de la biopolítica, los campos de concentración y los Estados totalitarios.

Como ya hemos señalado, la cuestión del derecho en las investigaciones de Michel Foucault requiere abordar, por un lado, la función genealógica de la historia del derecho en su ontología histórica de la Modernidad y, por otro, las nociones de derecho de los gobernados y de nuevo derecho, de las que no nos hemos ocupado aquí. En ese tratado acerca de la policía moderna -que consideramos el marco de referencia indispensable para abordar la relación entre derecho y Modernidad en el pensamiento foucaultiano- y en su analítica de la racionalidad política liberal de los siglos XVII y XVIII, que han sido el objeto de nuestro trabajo, las nociones de golpe de Estado permanente y de heterogeneidad entre las diferentes expresiones jurídicas nos muestran cómo, más que abandonarla, Foucault ha extendido la cuestión del derecho y su relación con la vida más allá de la perspectiva que nos brindan las nociones de excepción soberana y de vida desnuda, de captura y de culpa. Procediendo de este modo, como lo muestra su insistencia en la antinomia característica de la racionalidad política moderna, sin que ello le impida vincular el modo en que el Estado moderno se hace cargo de la vida de los individuos y de las poblaciones con los campos de concentración y los Estados totalitarios, es decir, con la posibilidad de una tanatopolítica.

Pero ni la excepción soberana, ni la producción de vida desnuda y, consecuentemente, tampoco el paradigma de los campos de concentración agotan o dominan las investigaciones foucaultianas sobre la racionalidad política moderna. A Foucault le interesa explicar, en efecto, cómo y según qué modalidades ha sido posible una biopolítica, con el sentido positivo que tiene este término en sus trabajos, es decir, como mejora y fortalecimiento de la vida, y no sólo una tanatopolítica. De este modo, allí donde en las investigaciones agambenianas nos encontramos con las nociones de captura y de culpa; en los análisis foucaultianos, hallamos, por un lado, los conceptos de integración 
marginalista y de utilitarismo político y, por otro, en ambos casos en su dimensión afirmativa, el "vivir y el más que vivir" y la vida expuesta al riesgo.

Por último, vale la pena subrayar que, más allá de las modalidades de una biopolítica, entendida como mejora y fortalecimiento de la vida, y de una tanatopolítica, entendida como producción de vida desnuda, los principios de integración marginalista y de utilitarismo político también nos permiten pensar, como ya señalamos, la posibilidad de una vida rechazada hacia la muerte, que resulta ser, finalmente, más desnuda que la agambeniana nuda vita.

\section{Referencias bibliográficas}

- Agamben, Giorgio, Che cos'è un dispositivo?, Roma, Nottetempo, 2006.

- Agamben, Giorgio, Homo sacer. Edirione integrale, Macerata, Quodlibet, 2018.

- Agamben, Giorgio, Homo sacer. Il potere sovrano e la nuda vita, Turín, Einaudi, 1995.

- Agamben, Giorgio, Il linguaggio e la morte, Turín, Einaudi, 1982.

- Agamben, Giorgio, Il Regno e la Gloria, Piacenza, Neri Pozza, 2007.

- Agamben, Giorgio, Infañia e storia, Turín, Einaudi, 1978.

- Agamben, Giorgio, Karman: Breve trattato sull'azione, la colpa e il gesto, Turín, Bollati Boringhieri, 2017.

- Agamben, Giorgio, Signatura rerum. Sul metodo, Turín, Bollati Boringhieri, 2008

- Benveniste, Émile, Le Vocabulaire des institutions indoeuropéenes, París, 1969.

- Castro, Edgardo, "La noción de policía en los trabajos de Michel Foucault: objeto, límites, antinomias", Anuario Colombiano de Historia Social y de la Cultura, 46, 2 (2019), pp. 185-206.

- Denis, Vincent, "L'histoire de la police après Foucault. Un parcours historien", Revue d'bistoire moderne et contemporaine, t. 60e, No 4/4 bis (2013), pp. 139-155.

- Dreyfus, Hubert y Rabinow, Paul, Michel Foucault: Beyond structuralism and hermeneutics, Brighton, Harvester Press, 1982. 
- Ewald, François, “Norms, Discipline, and the Law”, Representations, 30 (1990), pp. 138-161.

- Foucault, Michel, "Il faut défendre la société". Cours au collège de France. 1976, París, Gallimard-Seuil, 1997.

- Foucault, Michel, Dits et écrits, París, Gallimard, 1994

- Foucault, Michel, Histoire de la folie à l'âge classique, París, Gallimard, 1999.

- Foucault, Michel, La société punitive. Cours an Collège de France. 1972-1973, París, EHESS-Seuil-Gallimard, 2013.

- Foucault, Michel, La Volonté de savoir. Histoire de la sexualité 1, París, Gallimard, 1976.

- Foucault, Michel, Le Sonci de soi. Histoire de la sexualité 3, Paris, Gallimard, 1984.

- Foucault, Michel, Naissance de la biopolitique Cours au Collège de France. 19781979, París, Gallimard-Seuil, 2004.

- Foucault, Michel, Sécurité, territoire, population. Cours au Collège de France. 19771978, París, Gallimard-Seuil, 2004.

- Foucault, Michel, Théories et institutions pénales. Cours au Collège de France. 1971 1972, París, EHESS-Seuil-Gallimard, 2015.

- Frost, Tom, “Agamben's Sovereign Legalization of Foucault”, Oxford Journal of Legal Studies, Vol. 30, No. 3 (2010), pp. 545-577.

- Golder, Ben y Fitzpatrick, Peter, Foucault's law, Nueva York, Routledge, 2009.

- Hunt, Alan, "Foucault's Expulsion of Law. Toward a Retrieval", Law \& Social Inquiry, Vol. 17, No. 1 (1992), pp.1-38.

- Lawlor, Leonard y Nale, John, Cambridge Foucault Lexicon, Nueva York, Cambridge University Press, 2014.

- Moran, Brendan y Salzani, Carlo, Towards the critique of violence: Walter Benjamin and Giorgio Agamben, Londres-Nueva York: Bloomsbury, 2015.

- Napoli, Paolo, La naissance de la police moderne. Powvoir, normes, société, París, La Découverte, 2003. 
- Revel, Judith, Dictionnaire Foucault, París, Ellipses, 2008.

- Rose, Nikolas y Valverde, Mariana, "Governed by Law?", Social and Legal Studies 7, 4 (1998), pp. 541-551.

- Ruoff, Michael, Foucault Lexikon, Padeborn, Wilhem Fink, 2009.

Recibido: 02/2020. Aceptado: 05/2020 